\title{
A Turbo-detection Aided Serially Concatenated MPEG-4/TCM Videophone Transceiver
}

\author{
S. X. Ng, J. Y. Chung, F. Guo and L. Hanzo \\ School of ECS, University of Southampton, SO17 1BJ, UK. \\ Tel: +44-23-8059 3125, Fax: +44-23-8059 4508 \\ Email: $\{$ sxn,jyc00r,fg01r,lh\}@ecs.soton.ac.uk, http://www-mobile.ecs.soton.ac.uk
}

\begin{abstract}
A Turbo-detection aided serially concatenated inner Trellis Coded Modulation (TCM) scheme is combined with four different outer codes, namely with a Reversible Variable Length Code (RVLC), a Non-Systematic Convolutional (NSC) code a Recursive Systematic Convolutional (RSC) code or a Low Density Parity Check (LDPC) code. These four outer constituent codes are comparatively studied in the context of an MPEG4 videophone transceiver. These serially concatenated schemes are also compared to a stand-alone LDPC coded MPEG4 videophone system at the same effective overall coding rate. The performance of the proposed schemes is evaluated when communicating over uncorrelated Rayleigh fading channels. It was found that the serially concatenated TCM-NSC scheme was the most attractive one in terms of coding gain and decoding complexity among all the schemes considered in the context of the MPEG4 videophone transceiver. By contrast, the serially concatenated TCM-RSC scheme was found to attain the highest iteration gain among the schemes considered.
\end{abstract}

\section{MOTIVATION AND BACKGROUND}

The MPEG-4 standard $[1,2]$ offers a standardised framework for a whole range of multimedia applications. Examples include teleshopping, interactive TV, internet games, or mobile video communication. MPEG-4 integrates different types of multimedia data and services by the introduction of a so-called object-based approach for the description and coding of multimedia contents. The key functionalities of MPEG-4 include independent coding of objects in a video frame, the ability to interactively embed these video objects into a scene shown on the screen, the transmission of 3D scene descriptions, quality versus bitrate based temporal and spatial scalability and improved error resilience [3].

As the MPEG-4 standard targets a broader range of different applications and bitrates than the previously defined hybrid video coding standards such as MPEG-1, 2 or H.263, it employs a higher variety of different algorithms and coding modes. In the MPEG-4 coding algorithm a scene consists of one or more audio-visual objects potentially generated from multiple sources. A specific manifestation of a so-called video object layer is referred to as a video object plane (VOP) [2]. The individual VOPs delivered to an MPEG-4 decoder are allowed to have arbitrary shapes. The individual VOPs of an object may be transmitted separately from each other.

In MPEG-4 video coding, the algorithm employed for encoding natural video scenes is based on the classic block-based hybrid coding scheme [4], which is known from the well-established MPEG-1, 2 or H.263 codecs. However, these codecs were further developed in order to allow the encoding of arbitrarily shaped video objects. For

The financial support of both the EPSRC, Swindon UK and the EU under the auspices of the Phoenix project is gratefully acknowledged. employment in error-prone environments, error resilient features were introduced by several parts of the MPEG- 4 standards. This renders the MPEG-4 coding standard particularly suitable for wireless video telephony.

Trellis Coded Modulation (TCM) [5, 6] constitutes a bandwidthefficient joint channel coding and modulation scheme, which was originally designed for transmission over Additive White Gaussian Noise (AWGN) channels. More specifically, TCM schemes employ a non-binary Recursive Systematic Convolutional (RSC) code [5-7] and a set-partitioning scheme [5] labelled as mapper. In an effort to improve the performance of TCM when communicating over Rayleigh fading channels, In-phase (I) and Quadrature-phase (Q) interleaved TCM (IQ-TCM) schemes were proposed in $[8,9]$. Specifically, IQ-TCM benefits from additional signal-space diversity or IQdiversity, owing to the independent fading of the I and Q components, when communicating over Rayleigh fading channels.

Lossless Variable Length Codes (VLCs) constitute a family of low-complexity source compression schemes. Specifically, Huffman coding belongs to the family of VLCs that is capable of achieving optimum source compression. However, Huffman coding has no error correcting capability owing to its minimum free distance of unity among the legitimate codewords. However, VLCs can be designed for attaining a minimum free distance of higher than unity at the cost of a reduced source compression capability. In order to exploit the residual redundancy of VLCs, numerous trellis-based VLC decoding techniques have been proposed, such as the joint source/channel coding scheme of [10] and the joint source-coding, channel-coding and modulation scheme of [11], where a Reversible VLC (RVLC) [12] was invoked as the outer code and the associated bit-based trellis structure [13] was used for decoding. It has been shown in $[10,11]$ that RVLCs can be jointly utilised as the source compression scheme and as an outer channel code for providing a significant amount of coding gain, when they are jointly turbo-decoded with a serially concatenated inner channel code or TCM. Hence, a serially concatenated IQ-TCMRVLC scheme constitutes a good candidate for enhancing the performance of the MPEG4 videophone transceiver. More specifically, 4-bit video symbols can be created from the MPEG4-coded video bitstream and an RVLC can be designed based on the probability of occurrence of the $2^{4}=16$ possible values of the video symbols.

However the MPEG4-coded video bitstream may no longer be compressed by the RVLC scheme, if the 4-bit video symbols are equiprobable. In this scenario, maximal minimum distance Non-Systematic Convolutional (NSC) codes [14, p. 331] having a significantly lower number of trellis states compared to VLC having the same minimum distance may constitute a better candidate for concatenation with inner channel codes. On the other hand, the performance of the RSC code is poorer than that of the NSC code considered, when the number of iterations is low, since the minimum distance of the RSC code is lower than that of the NSC code. However, as a benefit of its recursive structure, the RSC code will outperform the NSC code, 


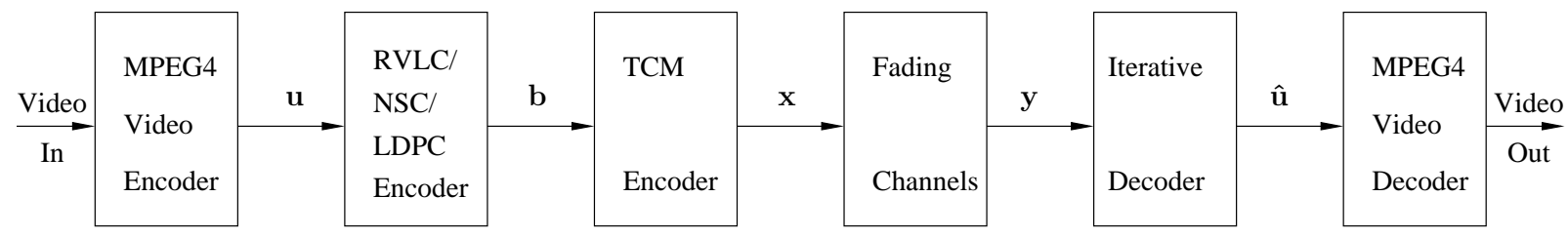

Figure 1: Block diagram of the serially concatenated MPEG4 IQ-TCM-RVLC/NSC/RSC/LDPC scheme. The notations $\mathbf{u}, \hat{\mathbf{u}}, \mathbf{b}, \mathbf{x}$ and $\mathbf{y}$ denote the vectors of the video symbols, the decoded estimates of the video symbols, the RVLC/NSC/RSC/LDPC outer encoded bits, the TCM symbols and the received symbols, respectively. The IQ channel interleaver and the bit-based interleaver between RVLC/NSC/RSC/LDPC and TCM are not shown for simplicity.

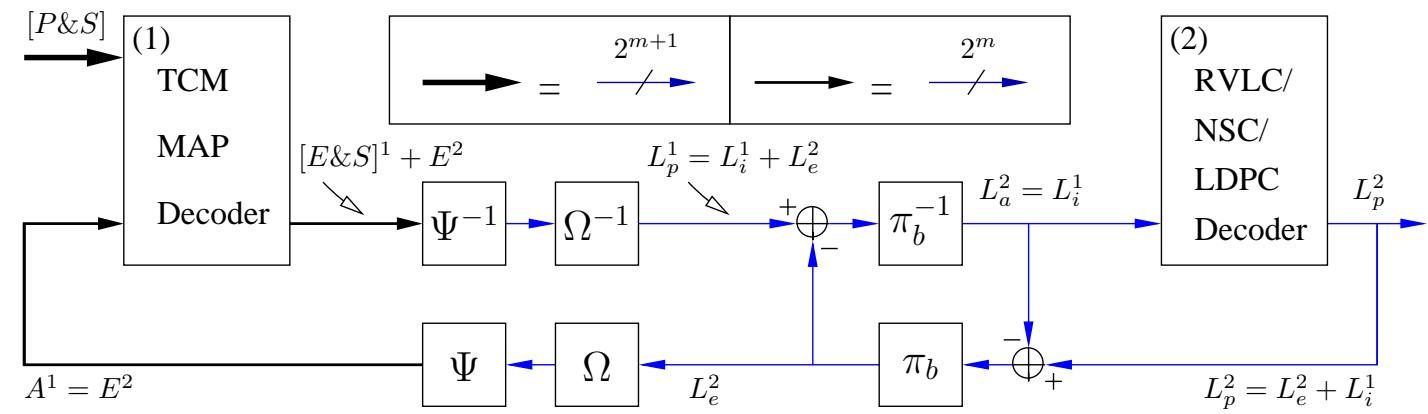

Figure 2: Block diagram of the IQ-TCM-RVLC/NSC/RSC/LDPC turbo detection scheme. The notations $\pi_{b}$ and $\pi_{b}^{-1}$ denote the bit-based interleaver and deinterleaver, respectively. Furthermore, $\Psi$ and $\Psi^{-1}$ denote LLR-to-symbol probability and symbol probability-to-LLR conversion, while $\Omega$ and $\Omega^{-1}$ denote the addition and deletion of the LLRs of the side information and dummy bits for the RVLC decoder.

when the number of iterations is sufficiently high. Furthermore, Low Density Parity Check (LDPC) codes [15] are capable of approaching the channel's capacity limit at the cost of a relatively high storage requirement and complexity.

In this contribution, we propose a variety of novel serially concatenated transceivers and comparatively study the performance of the concatenated IQ-TCM-RVLC, IQ-TCM-NSC, IQ-TCM-RSC and IQ-TCM-LDPC schemes as well as a stand-alone LDPC scheme, all having a similar coding rate, when communicating over Rayleigh fading channels.

\section{SYSTEM OVERVIEW}

The system block diagram of the MPEG4-based serially concatenated turbo scheme having IQ-TCM as the inner constituent code and RVLC, NSC, RSC or LDPC as the outer constituent code is shown in Figure 1. We fixed the transmission frame length to 2032 bits and 16level Quadrature Amplitude Modulation (16QAM) was employed. In the context of the IQ-TCM-RVLC scheme a low number of dummy bits was required for mapping the RVLC output bits to a constantlength bit sequence. The side information related to the number of RVLC output bits per transmission frame conveying the RVLCs is explicitly signalled to the decoder by repeating the related side information bits three times for the sake of employing majority logic based detection and the side information bits are then further protected by the TCM scheme. The coding rate of the RVLC scheme takes into account the rate loss due to the inclusion of the side information bits and the dummy bits.

The decoder structure of the IQ-TCM-RVLC/NSC/RSC/LDPC scheme is illustrated in Figure 2, where there are two constituent decoders, each labelled with a round-bracketed index. Symbol-based and bit-based MAP algorithms [6] operating in the logarithmic-domain are employed by the TCM/NSC/RSC decoder and by the RVLC de- coder, respectively, because no multiplication and division operations are required in the logarithmic-domain. By contrast, the Fast Fourier Transform (FFT) was invoked for LDPC decoding [16], which is less complex than the traditional belief-propagation based LDPC decoding method [17]. Note that the traditional belief-propagation algorithm can be implemented in the logarithmic domain according to the method proposed in [18] but it is only applicable for employment in binary LDPCs. By contrast, the less complex FFT-based LDPC decoding technique can be used for non-binary LDPCs, but it cannot operate in the logarithmic domain. Hence, multiplication and division operations are required by the FFT-based LDPC decoder instead of additions and subtractions.

The notations $P, S, A$ and $E$ in Figure 2 denote the logarithmicdomain probabilities of the parity information, the systematic information, the a priori information and the extrinsic information, respectively. The notations $L_{p}, L_{e}$ and $L_{i}$ denote the LogarithmicLikelihood Ratio (LLR) of the a posteriori, extrinsic and intrinsic information, respectively. The probabilities and LLRs associated with one of the two constituent decoders having a label of 1 and 2 are differentiated by the superscript of 1 and 2 . The logarithmic-domain symbol probabilities of the IQ-interleaved symbols are computed by the demodulator based on the approach of [9]. There are $2^{m+1}$ probabilities associated with an $(m+1)$-bit TCM-coded symbol, which have to be determined for the MAP decoder [6]. These probabilities are input to the TCM MAP decoder as $[P \& S]$, which indicates the inseparable nature of the parity and systematic information $[6,7]$. The TCM decoder's output is given by $[E \& S]^{1}+E^{2}$, where $[E \& S]$ can be referred to as intrinsic information, since it contains the extrinsic information provided by the TCM decoder itself as well as the systematic information at the demodulator's output $[P \& S]$. 


\begin{tabular}{|l|c|c|c|}
\hline Function & Number of terms & Multiplication & Addition \\
\hline F1) $\alpha_{t}(s)=\sum_{\text {all } \grave{s}} \gamma_{t}(\grave{s}, s) \cdot \alpha_{t-1}(\grave{s})$ & $S$ & $M S$ & $S(M-1)$ \\
F2) $\beta_{t-1}(\grave{s})=\sum_{\text {all } s} \beta_{t}(s) \cdot \gamma_{t}(\grave{s}, s)$ & $S$ & $M S$ & $S(M-1)$ \\
F3) $\gamma_{t}(\grave{s}, s)=\prod_{t, a} \cdot \eta_{t}(\grave{s}, s)$ & $M S$ & $M S$ & 0 \\
F4) $\gamma_{t}(\grave{s}, s)=\eta_{t}(\grave{s}, s)$ & $M S$ & 0 & 0 \\
F5) $\operatorname{Pr}\left\{u_{t}=a \mid \mathbf{y}\right\}=\sum_{\substack{(\grave{s}, s) \Rightarrow \\
u_{t}=a}} \beta_{t}(s) \cdot \alpha_{t-1}(\grave{s}) \cdot \gamma_{t}(\grave{s}, s)$ & $M$ & $2 M S$ & $M(S-1)$ \\
F6) $\operatorname{Pr}\left\{c_{t}=b \mid \mathbf{y}\right\}=\sum_{\substack{(\grave{s}, s) \Rightarrow \\
c_{t}=b}} \beta_{t}(s) \cdot \alpha_{t-1}(\grave{s}) \cdot \gamma_{t}(\grave{s}, s)$ & $\bar{M}$ & $\bar{M}\left(2 S \frac{M}{M}\right)=2 M S$ & $\bar{M}\left(S \frac{M}{M}-1\right)$ \\
\hline
\end{tabular}

Table 1: The total number of mathematical operations required for computing all terms of F1 to F6 per MAP decoder stage.

\section{COMPLEXITY}

The a posteriori probability of an $m$-bit information symbol $u_{t}$ given the received sequence $\mathbf{y}=\left\{y_{0}, \ldots, y_{N-1}\right\}$ generated by $N$ number of transmitted symbols may be computed as [6]:

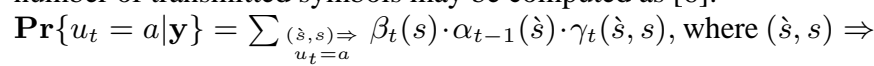
$u_{t}=a$ indicates the specific set of trellis transitions emerging from the previous trellis state $S_{t-1}=\grave{s}$ to the present state $S_{t}=s$ that can be encountered, when the $m$-bit information symbol is $u_{t}=a$, where $a$ is one of the legitimate $2^{m}$-ary information symbols. Similarly, the a posteriori probability of an $n$-bit coded symbol $c_{t}$ may be computed as:

$\operatorname{Pr}\left\{c_{t}=b \mid \mathbf{y}\right\}=\sum_{\substack{(\grave{s}, s) \Rightarrow \\ c_{t}=b}} \beta_{t}(s) \cdot \alpha_{t-1}(\grave{s}) \cdot \gamma_{t}(\grave{s}, s)$, where $(\grave{s}, s) \Rightarrow$ $c_{t}=b$ indicates the specific set of trellis transitions emerging from the previous trellis state $S_{t-1}=\grave{s}$ to the present state $S_{t}=s$ that can be encountered, when the $n$-bit coded symbol is $c_{t}=b$ and $b$ is one of the legitimate $2^{n}$-ary coded symbols. Furthermore, we have $\alpha_{t}(s)=\sum_{\text {all } \grave{s}} \gamma_{t}(\grave{s}, s) \cdot \alpha_{t-1}(\grave{s})$, which is the result of the MAP decoder's forward recursion [6], $\beta_{t-1}(\grave{s})=\sum_{\text {all } s} \beta_{t}(s) \cdot \gamma_{t}(\grave{s}, s)$ is that of the backward recursion [6] and $\gamma_{t}(\grave{s}, s)=\Pi_{t, a} \cdot \eta_{t}(\grave{s}, s)$ is the branch transition metric [6]. Finally, $\Pi_{t, a}$ is the a priori information regarding the information symbol $a$ and $\eta_{t}$ is the a priori information regarding the coded symbol, which can be obtained from the other decoder or from the demodulator and expressed as $\eta_{t}(\grave{s}, s)=$ $\exp \left(-\frac{\left|y_{t}-x\right|^{2}}{2 \sigma^{2}}\right)$ for AWGN channels. As usual, $x$ is the legitimate $2^{n}$-ary transmitted symbol corresponding to the information symbol $a$, while $y_{t}$ is the received signal at time $t$ and $\sigma^{2}$ is the noise's variance. Note that if $\Pi_{t, a}$ is not available, we have $\gamma_{t}(\grave{s}, s)=\eta_{t}(\grave{s}, s)$.

Table 1 quantifies the complexity of the MAP decoder for each trellis stage quantified in terms of the number of mathematical operations, where the coding rate is $R=m / n$ and the code memory is $\nu$. We also have $M=2^{m}, \bar{M}=2^{n}$ and $S=2^{\nu}$. Note that the bitbased RVLC trellis structure of [13] computes only the a posteriori information of the RVLC coded bits. Furthermore, not all states in the RVLC trellis have the same number of branches. Hence, we have to compute the average number of branches as $M=\hat{M} / S$, where $\hat{M}$ is the total number of branches at each trellis stage. Hence, we have $\bar{M}=M$ in Table 1 for the bit-based RVLC trellis. We also have to calculate the number of information bits $B$ per trellis/decoding stage, in order to quantify the complexity per information bit. Specifically, we have $B=R \cdot c b p s$, where $R$ is the coding rate and $c b p s$ represents the number of coded bits per symbol. Note that in the bit-based trellis structure of [13], the RVLC's cbps is equal to one.

Generally, the inner TCM decoder of Figure 2 has to compute only F1, F2, F3 and F5 of Table 1 for each iteration. By contrast, the outer NSC/RSC decoder of Figure 2 has to evaluate F1, F2, F4 and F6 for each iteration, while F1, F2, F4 and F5 only during the last iteration. Note that the bit-based outer RVLC decoder computes F1, F2, $\mathrm{F} 4$ and $\mathrm{F} 6$ for all iterations, but a sequence estimator is also invoked at the last iteration for generating the estimated information symbols based on the a posteriori probabilities of the coded bits. By contrast, the decoding complexity associated with each coded bit of the FFT-based binary LDPC codes may be calculated by appropriately modifying the approach of [16] as $10 c$ and $4 c$ number of multiplications and additions, respectively, where $c$ is the LDPC code's column weight. The number of information bits per binary LDPC decoding stage is given by $B=R$.

In the next section we will study the achievable performance of the proposed schemes using 16-level Quadrature Amplitude Modulation (16QAM) in the context of the $S=8$-state $R=3 / 4 \mathrm{TCM}$ scheme of [7, Table I], which protects all three information bits. Note that the 8 -state $R=3 / 4$ TCM scheme of [5, Table III] suffers from a relatively high error floor, when communicating over Rayleigh fading channels, since only two out of three information bits were protected. For the sake of extensive benchmarking we will concatenate the TCM scheme with four different serially concatenated outer encoders having an approximate code-rate of $R=3 / 4$. Specifically, the 8 -state $R=3 / 4$ NSC of $[14$, p. 331] having a minimum free distance of four, the 8 -state $R=3 / 4$ RSC of [7, Table I] having a minimum free distance of two and an approximately $R=0.777$ RVLC having a minimum free distance of two were invoked. Furthermore, two $c=3$ binary LDPC codes having coding rates of 0.777 and 0.576 were employed.

The associated number of trellis states of the RVLC having a minimum free distance of two was 29 in the context of the bit-based trellis structure of [13]. Note that in the RVLC scheme, there are only 15 trellis states that have two emerging trellis branches each, while the rest of the 14 states have only one trellis branch each, hence the average number of branches per trellis state is $M=(2 \times 15+14) / 29=$ 1.52. Table 2 summarises the decoding complexity per information

\begin{tabular}{|l|c|c|}
\hline Code & Multiplications/bit & Additions/bit \\
\hline TCM & $5 M S / B$ & $(S(3 M-2)-M) / B$ \\
RVLC/NSC/RSC & $4 M S / B$ & $(S(3 M-2)-M) / B$ \\
NSC/RSC (last iter.) & $4 M S / B$ & $(S(3 M-2)-M) / B$ \\
Binary LDPC & $10 c / B$ & $4 c / B$ \\
\hline
\end{tabular}

Table 2: Decoding complexity per information bit of the TCM, NSC, RSC, RVLC and LDPC schemes.

bit (and also per iteration in the case of LDPC codes) encountered for the constituent codes. Specifically, we have $S=8, M=8, \bar{M}=16$ and $B=3$ for the TCM, NSC and RSC schemes. By contrast, we have $S=29, M=\bar{M}=1.52$ and $B=0.777$ for the RVLC as well as $c=3$ and $B=0.777$ or $B=0.576$ for the LDPC codes.

\section{SIMULATION RESULTS}

In this section we evaluate the performance of the proposed MPEG4based video telephone schemes using the average video Peak Signal to Noise Ratio (PSNR) [4]. From Table 2, we can see that the RVLC decoder has the highest complexity owing to employing a higher number of trellis states than its NSC/RSC counterpart. By contrast, the LDPC schemes exhibit the lowest decoding complexity and hence they can 
afford invoking a certain number of internal iterations. Figures 3 to 7 depict the MPEG4 codec's video performance in conjunction with the serially concatenated TCM scheme having the NSC, RSC, RVLC or LDPC code as the outer constituent code as well as the stand-alone LDPC codes in the context of the average PSNR versus the Signal to Noise Ratio (SNR) per bit, namely $E_{b} / N_{0}$. The MPEG4 codec operated at 30 frames per second using the $(176 \times 144)$-pixel Quarter Common Intermediate Format Miss America video sequence, encoded at a bitrate of $69 \mathrm{kbps}$. The overall coding rate of the IQ-TCMRVLC, IQ-TCM-LDPC and LDPC schemes was approximately $R=$ 0.576 , while that of the IQ-TCM-NSC and IQ-TCM-RSC schemes was approximately $R=0.546$. Hence the effective throughput of the schemes studied was $\eta=R \log _{2}(16)=2.3$ Bits Per Symbol (BPS), except for the IQ-TCM-NSC and IQ-TCM-RSC schemes, which had an effective throughput of 2.24 BPS.

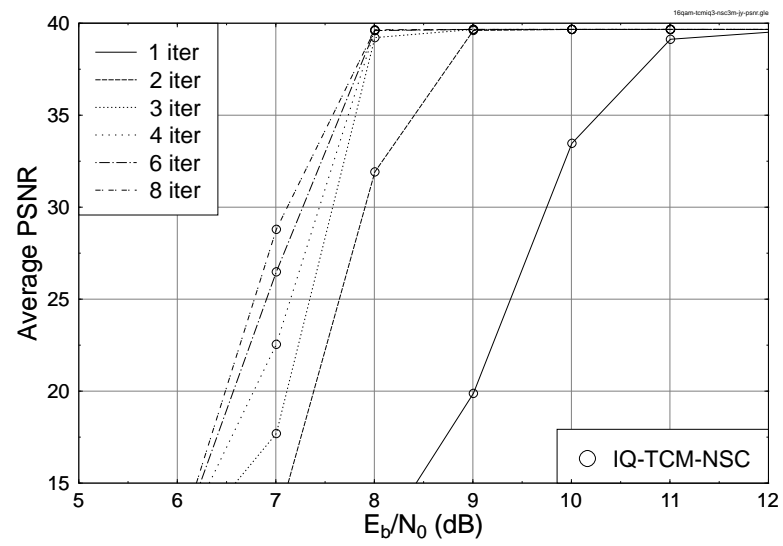

Figure 3: Average PSNR versus $E_{b} / N_{0}$ performance of the proposed 16QAM-based IQ-TCM-NSC assisted MPEG4 scheme, when communicating over Rayleigh fading channels. The effective throughput is $2.24 \mathrm{BPS}$.

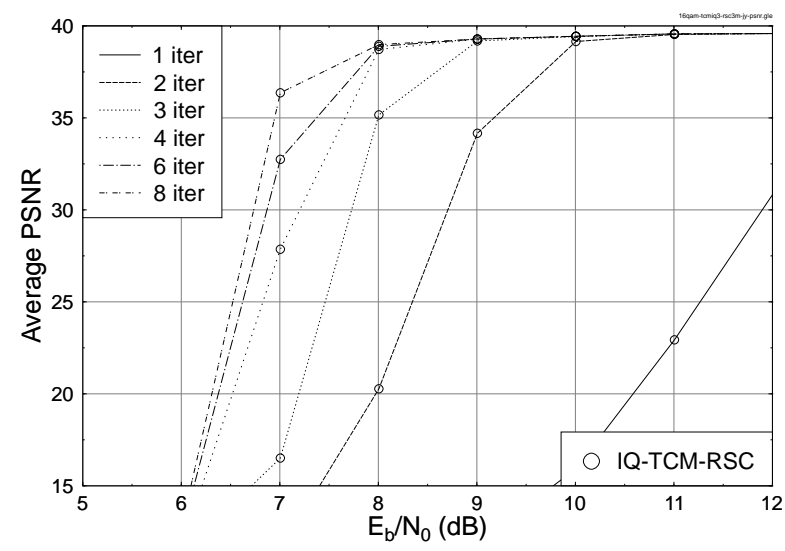

Figure 4: Average PSNR versus $E_{b} / N_{0}$ performance of the proposed 16QAM-based IQ-TCM-RSC assisted MPEG4 scheme, when communicating over Rayleigh fading channels. The effective throughput is $2.24 \mathrm{BPS}$.

It can be seen from Figures 5 to 7 that only the IQ-TCM-NSC, IQ-TCM-RSC and the stand-alone $R=0.576$ LDPC schemes managed to achieve an average PSNR in excess of $38 \mathrm{~dB}$ at $E_{b} / N_{0}=8 \mathrm{~dB}$. More specifically, the IQ-TCM-NSC scheme having three iterations requires $3 \times(106.7+85.3)=576$ multiplications/bit, while the

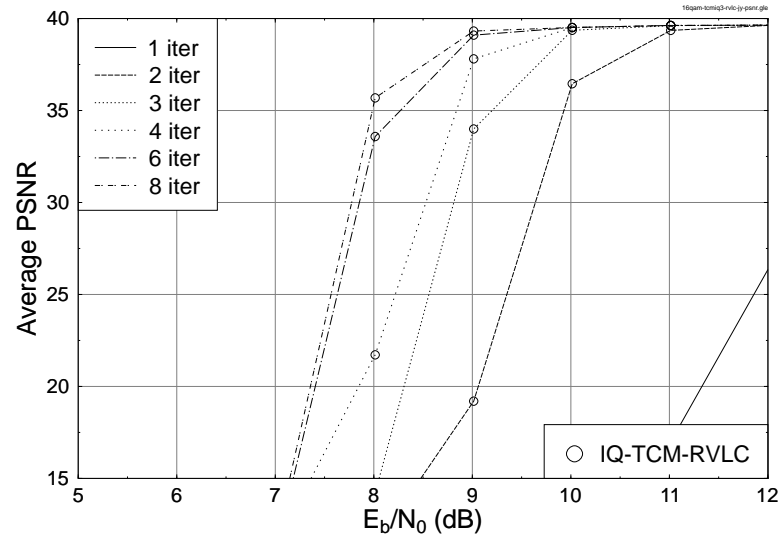

Figure 5: Average PSNR versus $E_{b} / N_{0}$ performance of the proposed 16QAM-based IQ-TCM-RVLC assisted MPEG4 scheme, when communicating over uncorrelated Rayleigh fading channels. The effective throughput was 2.30 BPS.

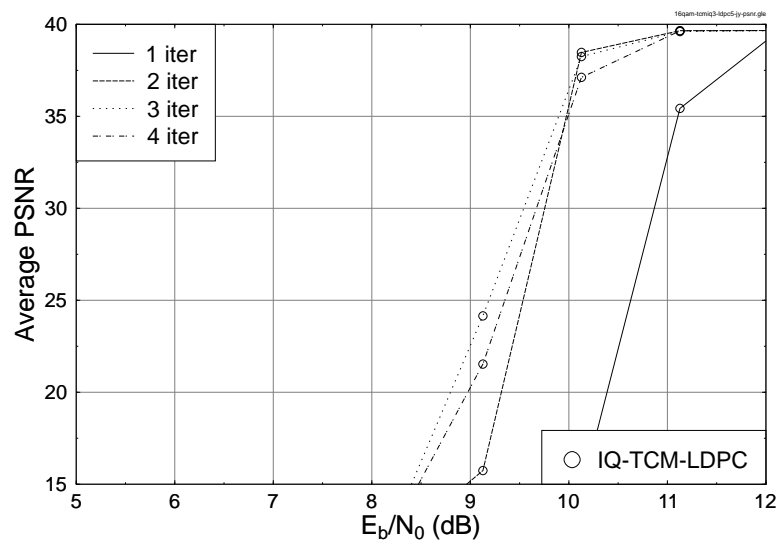

Figure 6: Average PSNR versus $E_{b} / N_{0}$ performance of the proposed 16QAM-based IQ-TCM-LDPC assisted MPEG4 scheme, when communicating over Rayleigh fading channels. The effective throughput is 2.30 BPS. The internal LDPC iterations is five and its coding rate is $R=0.777$.

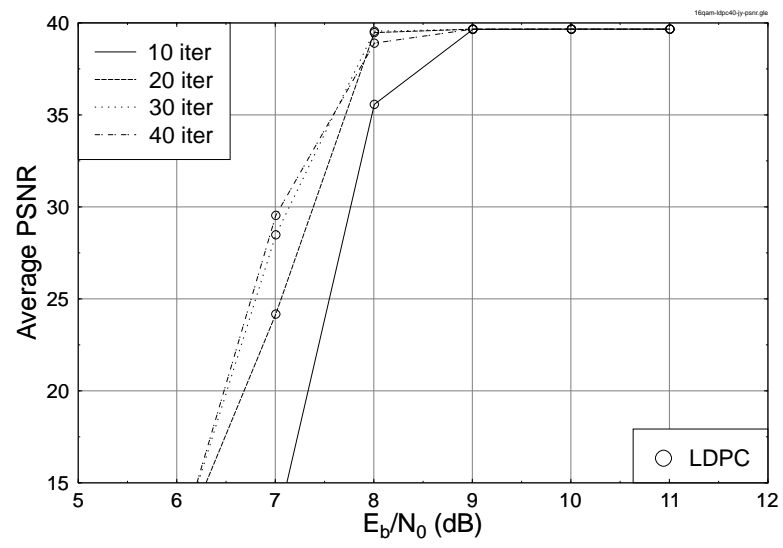

Figure 7: Average PSNR versus $E_{b} / N_{0}$ performance of the proposed 16QAM-based stand-alone LDPC assisted MPEG4 scheme, when communicating over Rayleigh fading channels. The effective throughput is 2.30 BPS. The coding rate of LDPC is $R=0.576$. 
IQ-TCM-RSC scheme having four iterations involves $4 \times(106.7+$ $85.3)=768$ multiplications/bit. Finally, the stand-alone $R=0.576$ LDPC code using 20 iterations requires $20 \times 52.1=1042$ multiplications/bit, when aiming for an average PSNR in excess of $38 \mathrm{~dB}$, as shown in Figures 3, 4 and 7. Therefore the IQ-TCM-NSC scheme has a lower decoding complexity than the IQ-TCM-RSC and the standalone LDPC schemes, while achieving an average PSNR of $38 \mathrm{~dB}$.

Note further that as predicted, IQ-TCM-RSC outperformed IQTCM-NSC after the fourth iteration as a benefit of the RSC code's recursive structure. More specifically, the IQ-TCM-RSC scheme managed to attain a PSNR in excess of $36 \mathrm{~dB}$ at $E_{b} / N_{0}=7 \mathrm{~dB}$, which is only $2.3 \mathrm{~dB}$ away from the Rayleigh fading channel capacity of 16QAM, which is $E_{b} / N_{0}=4.7 \mathrm{~dB}$, while maintaining an effective throughput of 2.24 BPS [19]. We also found during our studies that the MPEG4 output bits grouped as 4-bit symbols are fairly equiprobably distributed, hence the compression capability of the RVLCs was eroded. As a result, IQ-TCM-RVLC was outperformed by the IQTCM-NSC, IQ-TCM-RSC and LDPC schemes. Furthermore, the decoding complexity of IQ-TCM-RVLC is higher than that of IQ-TCMNSC and IQ-TCM-RSC, as we can see from Table 2.

On the other hand, the IQ-TCM-LDPC scheme of Figure 6 employs an 8-state IQ-TCM and the R=0.777 LDPC arrangement of Table 2. The number of LDPC iterations is five and hence the total number of multiplications per bit for the IQ-TCM-LDPC scheme amounts to $106.7+5 \times 38.6=299.7$ per outer iteration. As we can see from Figure 6, the LDPC scheme using five iterations is not well matched to the 8-state IQ-TCM inner code. More explicitly, the IQ-TCM-LDPC scheme benefits from no further iteration gains after the second outer iteration. After the second outer iteration the IQ-TCM-LDPC scheme required $E_{b} / N_{0} \approx 10 \mathrm{~dB}$ for maintaining PSNR=35 dB according to Figure 6, while the number of multiplications per bit required was $2 \times 299.7=599.4$. It is interesting to observe that the stand-alone $R=0.576$ LDPC scheme of Table 2 performed significantly better than the concatenated IQ-TCM-LDPC scheme, as it transpires from Figures 6 and 7. Specifically, the $R=0.576$ LDPC scheme employing 10 iterations has $52.1 \times 10=521$ multiplications/bit according to Table 2 and it requires $E_{b} / N_{0} \approx 8 \mathrm{~dB}$ for attaining PSNR=35 dB according to Figure 7. Hence the stand-alone LDPC scheme having 10 iterations requires an approximately $2 \mathrm{~dB}$ lower $E_{b} / N_{0}$ than the IQ-TCM-LDPC scheme at a comparable complexity and a similar effective throughput of 2.3 BPS.

\section{CONCLUSIONS}

In this contribution a range of serially concatenated IQ-TCM and RVLC/NSC/ RSC/LDPC schemes were studied and compared to a stand-alone LDPC code at a similar overall coding rate and throughput in the context of an MPEG4 video-telephone transceiver. It was shown that owing to the equiprobably distributed 4-bit MPEG4 output symbols, the compression capability of the RVLCs eroded and hence it was unable to further reduce the bitrate of the MPEG4 scheme. On the other hand, the stand-alone LDPC codec outperformed its IQ-TCM-LDPC counterpart, as shown in Figures 6 and 7. However, the IQ-TCM-NSC scheme was capable of attaining the same video performance as that of the IQ-TCM-NSC and LDPC codes at a lower complexity, when the number of turbo iterations invoked by the IQ-TCM-NSC/IQ-TCM-RSC scheme was low, as evidenced by Figures 3, Figure 4 and 7. By contrast, the IQ-TCM-RSC scheme was found to outperform the IQ-TCM-NSC scheme, when the number of iterations was sufficiently high. Hence, the IQ-TCM-NSC scheme was found to be the most beneficial scheme in assisting the MPEG4 video transceiver's operation, when a low decoding complexity was required. However, the IQ-TCM-RSC scheme constitutes the best design choice, when increasing the number of decoding iterations since a higher decoding complexity is affordable.

\section{REFERENCES}

[1] ISO/IEC JTC1/SC29/WG11, "Information Technology - Generic coding of Audio-visual Objects.," in Part 2: Visual. Draft ISO/IEC 14496-2 (MPEG-4), version 1, ISO/IEC, (Geneva), 1998.

[2] ISO/IEC JTC1/SC29/WG11 W2502 in ISO/IEC 14496-2. Final Draft International Standard. Part 2: Visual, (Atlantic City), 1998.

[3] B. Haskell, A. Puri, and L. Rabiner, "Image and Video Coding-Emerging Standards and beyond," vol. 8, pp. 814-837, November 1998.

[4] L. Hanzo, P.J. Cherriman and J. Street, Wireless Video Communications: Second to Third Generation Systems and Beyond. NJ, USA : IEEE Press., 2001.

[5] G. Ungerböck, "Channel Coding with Multilevel/Phase Signals," IEEE Transactions on Information Theory, vol. 28, pp. 55-67, January 1982.

[6] L. Hanzo, T. H. Liew and B. L. Yeap, Turbo Coding, Turbo Equalisation and Space Time Coding for Transmission over Wireless channels. New York, USA: John Wiley IEEE Press, 2002.

[7] P. Robertson, T. Wörz, "Bandwidth-efficient Turbo Trellis-coded Modulation Using Punctured Component Codes," IEEE Journal on Selected Areas in Communications, vol. 16, pp. 206-218, February 1998.

[8] B. D. Jelicic and S. Roy, "Design of Trellis Coded QAM for Flat fading and AWGN channels," IEEE Transactions on Vehicular Technology, vol. 44, pp. 192-201, February 1994.

[9] S. X. Ng and L. Hanzo, "Space-time IQ-interleaved TCM and TTCM for AWGN and Rayleigh fading Channels," IEE Electronics Letters, vol. 38, pp. 1553-1555, November 2002.

[10] R. Bauer, J. Hagenauer, "On Variable Length Codes for Iterative Source/Channel Decoding," in IEEE Data Compression Conference, (UT, USA), pp. 273-282, 27-29 March 2001.

[11] S. X. Ng, F. Guo, J. Wang, L-L. Yang and L. Hanzo, "Joint Sourcecoding, Channel-coding and Modulation schemes for AWGN and Rayleigh Fading Channels," IEE Electronics Letters, vol. 39, pp. 1259-1261, August 2003.

[12] Y. Takishima, M. Wada and H. Murakami, "Reversible Variable Length Codes," IEEE Transactions on Communications, vol. COM-43, no. 2/3/4, pp. 158-162, 1995.

[13] V. B. Balakirsky, "Joint Source-channel Coding with Variable Length Codes," in IEEE International Symposium on Information Theory, (Ulm, Germany), p. 419, 29 June - 4 July 1997.

[14] S. Lin and D. J. Costello, Jr, Error Control Coding: Fundamentals and Applications. Inc. Englewood Cliffs, New Jersey 07632: Prentice-Hall, 1983.

[15] R. Gallager, "Low Density Parity Check Codes," IRE Transactions On Information Theory, 1962.

[16] M.C. Davey, "Error-Correction using Low Density Parity Check Codes," Ph.D thesis, University of Cambridge,UK, 1999.

[17] T. Richardson, R. Urbanke, "The Capacity of Low-Density Parity Check Codes Under Message-Passing Decoding," IEEE Transaction on Information Theory, pp. 599-618, Feb. 2001.

[18] X.-Y. Hu, E. Eleftheriou, D,-M. Arnold and A. Dholakia, "Efficient implementations of the sum-product algorithm for decoding LDPC codes," IEEE GLOBECOM, vol. 2, pp. 1036-1036E, November 2001.

[19] S. X. Ng and L. Hanzo, "On the MIMO Channel Capacity of MultiDimensional Signal Sets," IEEE Vehicular Technology Conference, 2629 September 2004 